\title{
Origin of multiple periodicities in the Fourier power spectra of the Plasmodium falciparum genome
}

\author{
Miriam CS Nunes ${ }^{1}$, Elizabeth F Wanner ${ }^{2}$, Gerald Weber ${ }^{3 *}$ \\ From 6th International Conference of the Brazilian Association for Bioinformatics and Computational Biology \\ (X-meeting 2010) \\ Ouro Preto, Brazil. 15-18 November 2010
}

\begin{abstract}
Background: Fourier transforms and their associated power spectra are used for detecting periodicities and protein-coding genes and is generally regarded as a well established technique. Many of the periodicities which have been found with this method are quite well understood such as the periodicity of 3 nt which is associated to codon usage. But what is the origin of the peculiar frequency multiples $k / 21$ which were reported for a tiny section of chromosome 2 in $P$. falciparum? Are these present in other chromosomes and perhaps in related organisms? And how should we interpret fractional periodicities in genomes?

Results: We applied the binary indicator power spectrum to all chromosomes of $P$. falciparum, and found that the frequency overtones $k / 21$ are present only in non-coding sections. We did not find such frequency overtones in any other related genomes. Furthermore, the frequency overtones were identified as artifacts of the way the genome is encoded into a numerical sequence, that is, they are frequency aliases. By choosing a different way to encode the sequence the overtones do not appear. In view of these results, we revisited early applications of this technique to proteins where frequency overtones were reported.

Conclusions: Some authors hinted recently at the possibility of mapping artifacts and frequency aliases in power spectra. However, in the case of $P$. falciparum the frequency aliases are particularly strong and can mask the 1/3 frequency which is used for gene detecting. This shows that albeit being a well known technique, with a long history of application in proteins, few researchers seem to be aware of the problems represented by frequency aliases.
\end{abstract}

\section{Background}

The detection and analysis of repetitions in genomes is one of the most recurrent problems in computational biology. It is of technological importance since it imposes significant limitations to next-generation sequencing technologies [1] and is related to numerous properties of the genome [2-5]. As a consequence it has sparked many approaches to detect and visualise genomic repeats [6]. Discrete Fourier transform (DFT) is one

\footnotetext{
* Correspondence: gweberbh@gmail.com

${ }^{3}$ Department of Physics, Federal University of Minas Gerais, 31270-901 Belo Horizonte, MG, Brazil

Full list of author information is available at the end of the article
}

of this approaches, which is employed for the detection of genome wide non-exact periodic structures such as tandem repeats. In essence, the symbolic genome sequence is translated into numeric series which are then handled as a biological signal. The method was originally proposed for detecting periodicities in proteins [7-11] but has since gained popularity for genomes [12-18]. The genome of Plasmodium falciparum, the malaria parasite, is quite remarkable: it has an unusually high AT-content (80\% or more) [19] and lacks identified transposable elements [20]. Sharma et al. [21] reported a complete frequency comb of all overtones of the basic frequency $1 / 21 \mathrm{nt}^{-1}$ for chromosome 2 of P. falciparum,
Ciomed Central

() 2011 Nunes et al; licensee BioMed Central Ltd. This is an open access article distributed under the terms of the Creative Commons Attribution License (http://creativecommons.org/licenses/by/2.0), which permits unrestricted use, distribution, and reproduction in any medium, provided the original work is properly cited. 
that is, all frequencies of type $k / 21 \mathrm{nt}^{-1}$ with $k=1,2, \ldots, 10$. Could this be yet another peculiarity of the $P$. falciparum genome? Such a frequency comb envisages several questions such as its biological origin and function, that were not addressed by Sharma et al. [21] and have remained unanswered, to the best of our knowledge. Additionally, it also gives rise to an important conceptual question: how should we understand fractional periodicities such as $21 / 2$ or 21/4 given that there are no fractional nucleotides in our sequence? Or could it be that these multiples are so called frequency aliases $[22,23]$ ? In this work we analyse these questions more closely. First, we apply the Fourier transform to all chromosomes of P. falciparum and confirm that the $1 / 21 \mathrm{nt}^{-1}$ overtone frequency comb is found in all but one chromosomes and present its location in the genome. But perhaps most importantly, we have established that this frequency comb is indeed composed of frequency aliases, and as such is an artifact of the way the genome is converted from a symbolic into a numeric sequence. While this resolves our current conceptual problem, it poses an important warning for the blind use of this technique, especially if used to detect genes. Should one of the frequency multiples coincide with the $1 / 3$ frequency this could lead to important problems with the detection of coding regions [18,24-27]. We show that this is indeed the case for the 7/ 21 frequency of $P$. falciparum, which could cause false positive candidates for coding regions.

\section{Results and Discussion}

In Fig. 1 we present the binary indicator power spectra for all chromosomes of $P$. falciparum separated by coding
(CDS) and non-coding (non-CDS) regions. The $k / 21$ frequency comb can be seen only for the non-coding sections of chromosomes 1-13 in Fig. 1b and is notably absent in chromosome 14. No chromosomes show this particular frequency comb in the coding section, Fig. 1a. However, chromosome 5 displays a slightly different frequency comb of $k / 18$. Strong $1 / 3 \mathrm{nt}^{-1}$ peaks in Fig. $1 \mathrm{a}$ and the lack thereof in Fig. 1b confirms that this genome appears to be well annotated [18,24-27].

Further refining the origin of the $k / 21$ frequency comb shows that it comes mostly from regions at the extremes of the chromosomes, as shown in Fig. 2a, where we indicate the position dependent power spectra intensities for each of the $k / 21$ overtones in chromosome 1 . In contrast, the $k / 18$ frequency comb originates from various regions of the genome as one would expect for CDS regions.

To identify the actual sequences involved in the $k / 21$ frequency overtones, we extracted the first and last $4000 \mathrm{nt}$ of each chromosome and analysed the repetitions with Tandem Repeats Finder [28]. The consensus sequences are shown in table 1, which indicates single occurrences of dimers CC or GG. For instance, chromosome 1 has one GG dimer, which is the only occurrence of guanine in this sequence. Therefore, this dimer will repeat with frequency $1 / 21 \mathrm{nt}^{-1}$ and explains the fundamental frequency $1 / 21$ $\mathrm{nt}^{-1}$ which we observe in Fig. $1 \mathrm{~b}$.

However, we still do not know where the multiples of this frequency originate. The answer to this lies in a closer inspection of the repeated consensus sequences in table 1. Let's take sequence TAAGACCTATGTTAGTAAAG for chromosome 6 which has a double

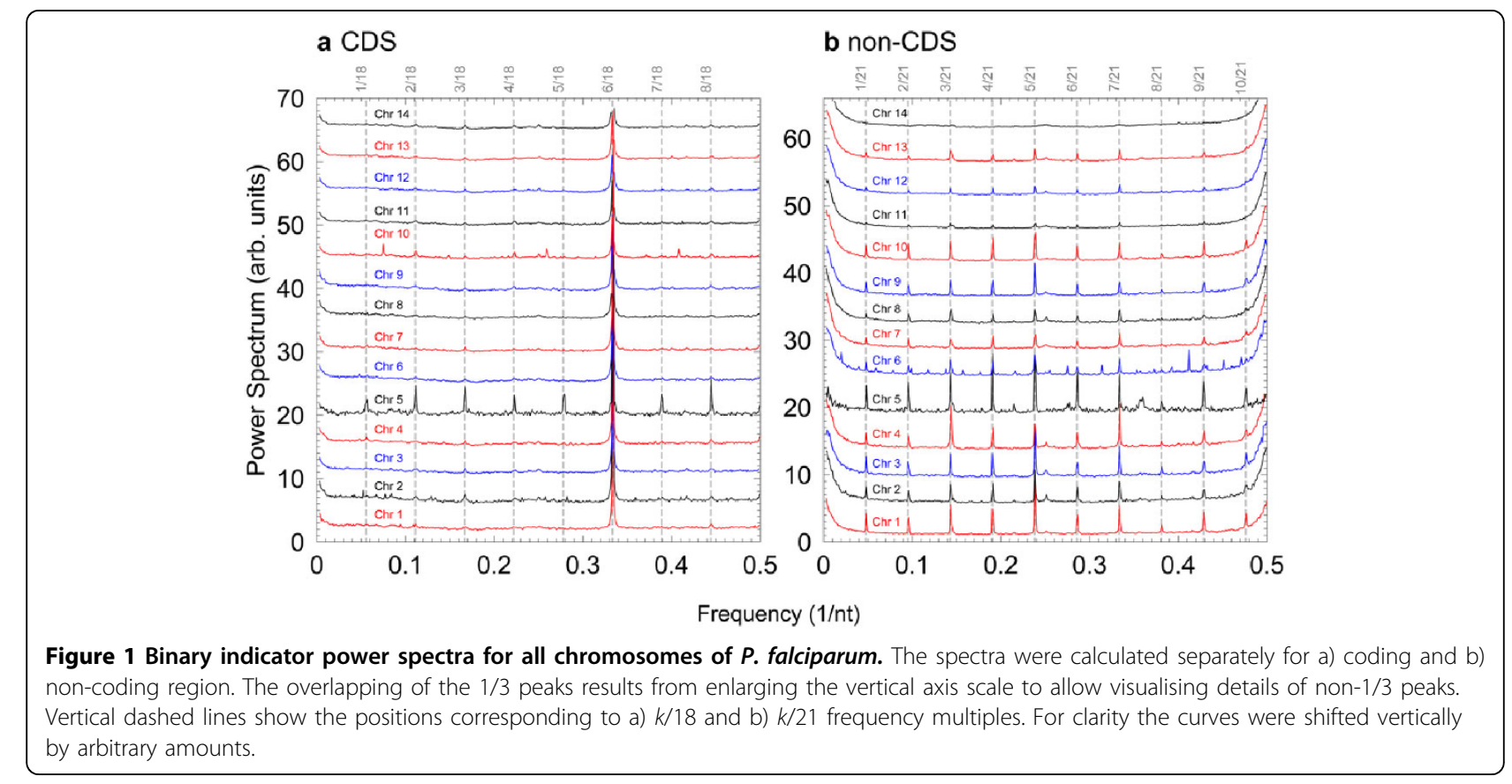




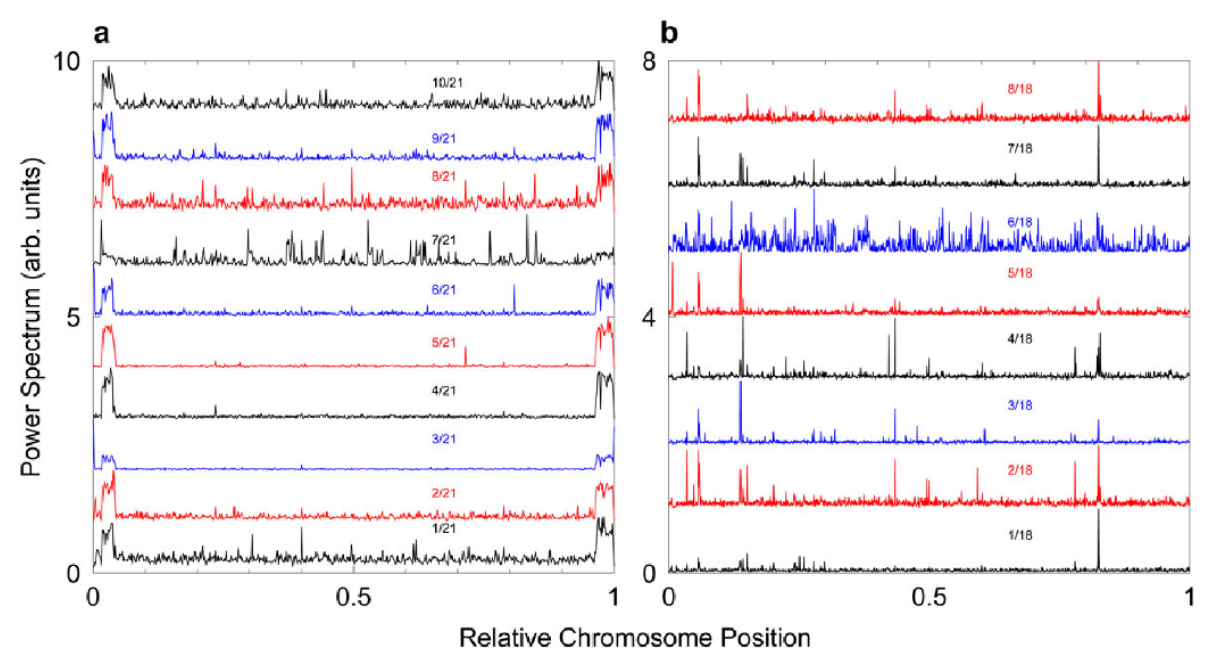

Figure 2 Position-dependent power spectra of $\boldsymbol{P}$. falciparum. Part a) is for chromosome 1 and its $k / 21$ frequency multiples and b) is for chromosome 5 and $k / 18$ frequency multiples. A sliding window of $\boldsymbol{w}=1000 \mathrm{nt}$ was used. For clarity the curves were shifted vertically by arbitrary amounts.

cytosine. The four binary indicator sequences which result from converting the symbolic sequence are shown in table 2. The binary sequence for cytosine in table 2 displays two consecutive ones followed by zeroes only.
In other words, we have two digits one followed by 19 zeroes, repeated hundreds of times. This is essentially analogous to a Fourier integral of a train of Kronecker delta functions, that is, a sequence of pulses with period

Table 1 Consensus sequences for periodicity $21 \mathrm{nt}$.

\begin{tabular}{|c|c|c|c|}
\hline Chromosome & Relative Position & Consensus sequence & Repetitions \\
\hline \multirow[t]{2}{*}{1} & $0.0179-0.0383$ & CTTAACTAACATAGGTCTTAA & 622.8 \\
\hline & $0.965-0.997$ & AGTTAGTTAAGTTAAGACCCTA & 994.3 \\
\hline \multirow[t]{2}{*}{2} & $0.0146-0.0223$ & ACTAACTTAGGTCTTAACTTA & 348.2 \\
\hline & $0.981-0.986$ & TTAGTTAAGTTAAGACCTAA ${ }^{\dagger}$ & 264.3 \\
\hline \multirow[t]{4}{*}{3} & $0.0126-0.0236$ & GGTCTTACTTCACTAACATA $^{\dagger}$ & 552.2 \\
\hline & $0.0157-0.0234$ & ATAGGTCTTAACTTAACTAAC & 417.6 \\
\hline & $0.0121-0.0283$ & TAGGTCTTAATTAACTAACT $^{\dagger}$ & 817.5 \\
\hline & $0.986-0.988$ & TAAGACCTAAGTTAGTGAAGT & 78.6 \\
\hline \multirow[t]{2}{*}{4} & $0.0139-0.0221$ & AGGTCTTACTTCACTAACTT ${ }^{\dagger}$ & 472.4 \\
\hline & $0.986-0.991$ & TTAGTTAAGTTAAGACCTAAG & 238.2 \\
\hline 5 & $0.0087-0.0129$ & ACTAACATAGGTCTTACTTC ${ }^{\dagger}$ & 269.8 \\
\hline \multirow[t]{2}{*}{6} & $0.977-0.979$ & TAAGACCTATGTTAGTAAAG $^{\dagger}$ & 152.7 \\
\hline & $0.982-0.990$ & AAAGTTAAGACCTAAGTTAGT & 581.6 \\
\hline 7 & $0.991-0.999$ & TTAAGACCTAAGTTAGTGAAG & 543.8 \\
\hline \multirow[t]{2}{*}{8} & $0.0077-0.0119$ & ACTAGGTCTTAACTTAACTA ${ }^{\dagger}$ & 269.6 \\
\hline & $0.988-0.991$ & AAGACCTAAGTTAGTAAGTT ${ }^{\dagger}$ & 185.4 \\
\hline \multirow[t]{2}{*}{9} & $0.978-0.985$ & GACCTATGTTAGTAAAGTAA ${ }^{\dagger}$ & 551.6 \\
\hline & $0.987-0.993$ & TAAGACCTAAGTTAGTGAAG ${ }^{\dagger}$ & 413.4 \\
\hline \multirow[t]{2}{*}{10} & $0.0073-0.0116$ & TAGGTCTTACTTTAACTAACT & 344.4 \\
\hline & $0.981-0.990$ & GACCTATATTAGTTAAGTTAA & 737.5 \\
\hline 11 & $0.0072-0.0093$ & TTACTAACATAGGTCTTAAC ${ }^{\dagger}$ & 202.3 \\
\hline \multirow[t]{2}{*}{12} & $0.0049-0.0055$ & TAACTTAGGTCTTAACTTCAC & 67.5 \\
\hline & $0.991-0.995$ & TATGTTAGTTAAGTTAAGACC & 354.0 \\
\hline \multirow[t]{3}{*}{13} & $0.0049-0.0069$ & ACTAACATAGGTCTTACTTC $\mathrm{C}^{\dagger}$ & 269.8 \\
\hline & $0.990-0.991$ & AGTAAGACCTTAGTTAGTGA ${ }^{+}$ & 192.5 \\
\hline & $0.991-0.993$ & GACCTATGTTAGTGAAGTTAA & 345.2 \\
\hline
\end{tabular}

Main tandem repetitions of periodicity $21 \mathrm{nt}$ found in the $P$. falciparum genome, except for chromosome 14 from which no such periodicities were detected. ${ }^{\dagger}$ Some consensus sequences provided by Tandem Repeats Finder [28] are of size $20 \mathrm{nt}$, yet they all correspond to the periodicity of $21 \mathrm{nt}$. 
Table 2 Example of sequence binary mapping.

\begin{tabular}{lccccccccccccccccccccccc}
\hline & $\mathrm{T}$ & $\mathrm{A}$ & $\mathrm{A}$ & $\mathrm{G}$ & $\mathrm{A}$ & $\mathrm{C}$ & $\mathrm{C}$ & $\mathrm{T}$ & $\mathrm{A}$ & $\mathrm{T}$ & $\mathrm{G}$ & $\mathrm{T}$ & $\mathrm{T}$ & $\mathrm{A}$ & $\mathrm{G}$ & $\mathrm{T}$ & $\mathrm{A}$ & $\mathrm{A}$ & $\mathrm{A}$ & $\mathrm{G}$ \\
\hline$n$ & 0 & 1 & 2 & 3 & 4 & 5 & 6 & 7 & 8 & 9 & 10 & 11 & 12 & 13 & 14 & 15 & 16 & 17 & 18 & 19 \\
$u_{A, n}$ & 0 & 1 & 1 & 0 & 1 & 0 & 0 & 0 & 1 & 0 & 0 & 0 & 0 & 1 & 0 & 0 & 1 & 1 & 1 & 0 \\
$u_{C, n}$ & 0 & 0 & 0 & 0 & 0 & 1 & 1 & 0 & 0 & 0 & 0 & 0 & 0 & 0 & 0 & 0 & 0 & 0 & 0 & 0 \\
$u_{G, n}$ & 0 & 0 & 0 & 1 & 0 & 0 & 0 & 0 & 0 & 0 & 1 & 0 & 0 & 0 & 1 & 0 & 0 & 0 & 0 & 1 \\
$u_{T, n}$ & 1 & 0 & 0 & 0 & 0 & 0 & 0 & 1 & 0 & 1 & 0 & 1 & 1 & 0 & 0 & 1 & 0 & 0 & 0 & 0 \\
\hline
\end{tabular}

$T$, which can be shown to result in another train of Kronecker delta functions with period $1 / T$ in frequency space [29]. Therefore, the overtones $k / 21$ do not correspond to real periods $21 / k$ but are just artifacts of the way the symbolic sequence was mapped into binary sequences. Therefore, the frequencies $2 / 21,3 / 21 \ldots, 10 /$ 21 are frequency aliases of the frequency $1 / 21[22,30]$.

In Fig. 3 we illustrated this effect by building an artificial sequence made of 80 repetitions of the basic unit of size 21 nt containing one or more consecutive cytosines. For ten cytosines we obtain exactly one peak at $1 / 21 \mathrm{nt}^{-}$ ${ }^{1}$ as expected. However, as we gradually reduce the number of consecutive cytosines, while maintaining the size of the repeating unit constant to $21 \mathrm{nt}$, we also obtain more of the $k / 21$ overtones. Eventually, for one or two cytosines, all overtones $k / 21$ are present, that is, we obtain all fractional periodicities although only one cytosine repeats every 21 positions.

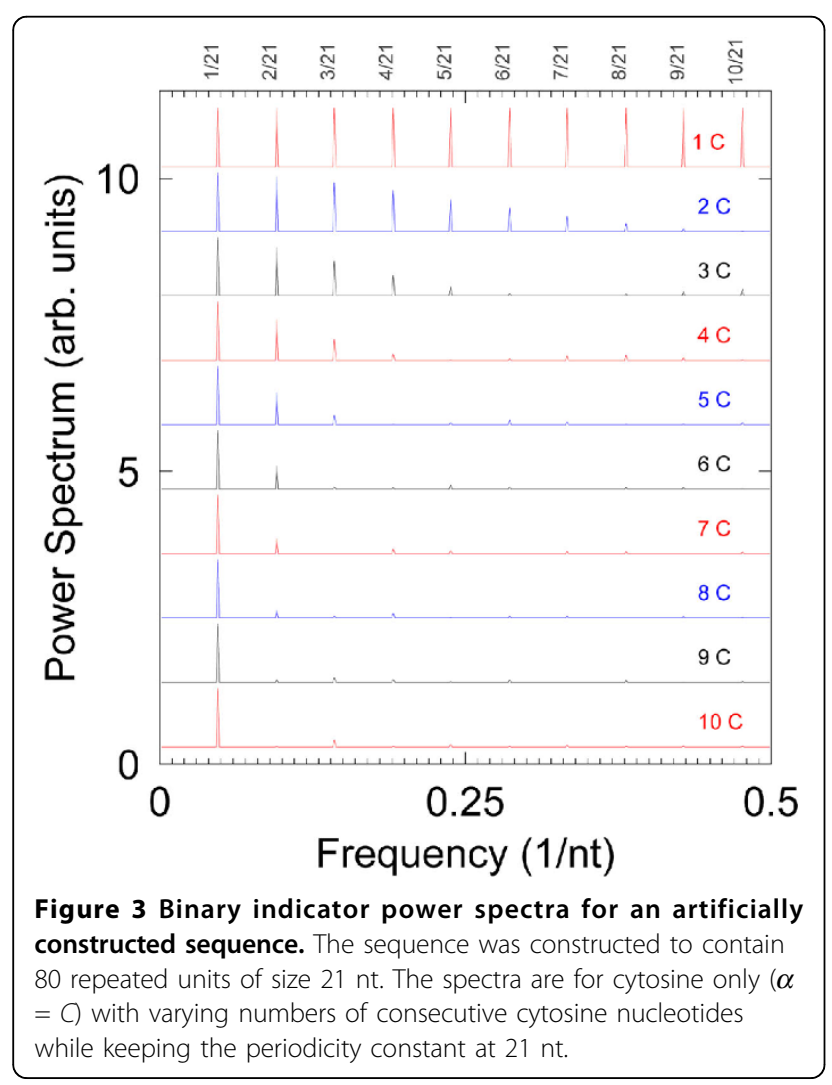

One simple way to verify if these overtones are artifacts of the numerical mapping is to change the mapping. As an example, we computed the power spectra using DNA flexibility mapping, as detailed in Methods section. In this case the symbolic sequence is converted into a single numerical sequence and instead of using only zeroes and ones we have now ten different numerical values. This particular mapping was selected because converting di-nucleotides allows us to choose over a larger set of numerical values resulting into an overall smoother numerical profile along the sequence. Therefore the occurrence of delta-like structures in the numerical sequence should be less likely. The power spectra for both coding and non-coding sections of $P$. falciparum are shown in Fig. 4. As expected, the frequency overtones are now barely noticeable for a few chromosomes only. Most chromosomes show little or no overtones at all. We have established that the occurrence of overtones is the result of one or two isolated nucleotides surrounded by a large number of different nucleotides and very frequently repeated. But what is the minimal size of the repeating unit for which overtones may start to appear? We carried out numerical tests using a simple repeating unit $\mathrm{CA}_{n}$ with $n=1,2$, ..., 9, corresponding to a periodicity of $p=n+1$ (data not shown), and overtones were present in all cases.

\section{Conclusions}

It was suggested that the mapping of the symbolic sequence into one or more numeric sequences could give rise to artifacts when signal processing techniques are applied [31]. In this case, the frequency comb results as an artifact of dividing one symbolic sequence into four separate sequences, that is, it is an artifact of the multichannel analysis. Indeed, for proteins fractional periodicities were already observed in the pioneering work by McLachlan and Stewart [7,9]. The sequence mapping used some amino acid properties and is similar to the flexibility mapping outlined in the Methods section. In this case, the authors merely state that the periodicity does not need to be an integer value $[7,9]$. While non-integral periods for certain mappings may be plausible, multiple overtones are harder to explain in this way. Subsequent works, such as McLachlan et al. [32], explicitly use the multichannel mapping and clearly 


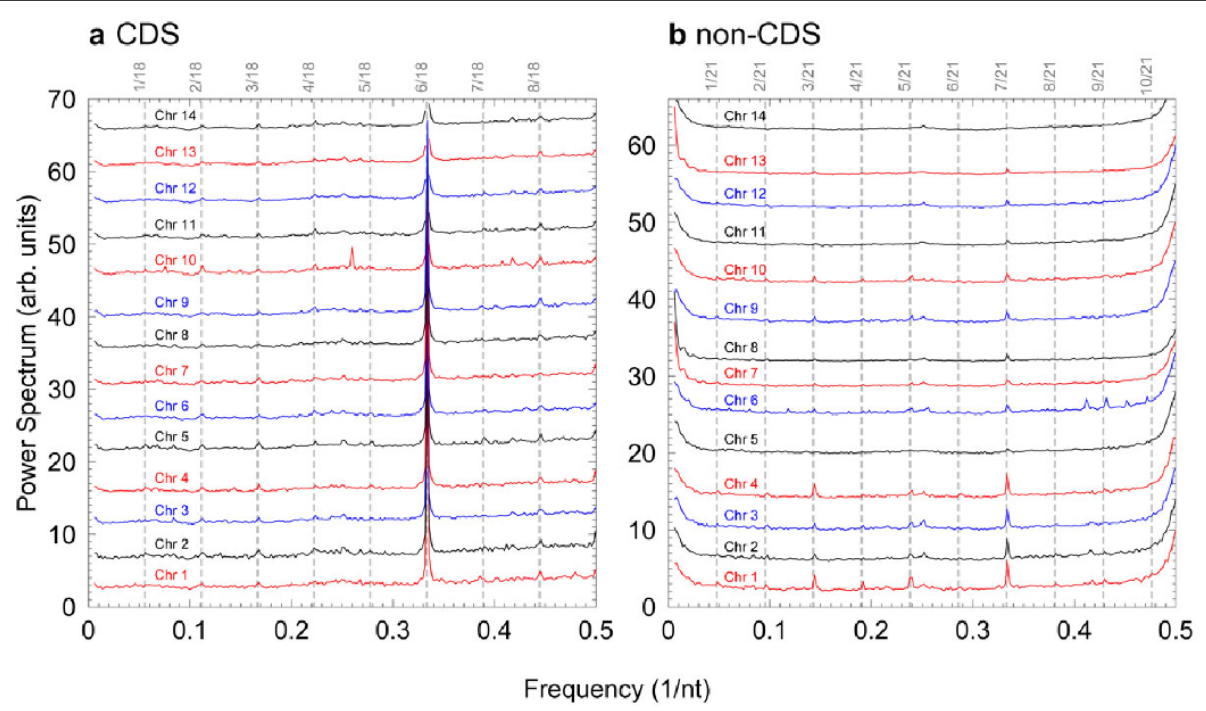

Figure 4 Flexibility power spectra for each chromosome of $\boldsymbol{P}$. falciparum. Part a) is for coding regions and b) for non-coding regions. Also shown are the vertical dashed lines for the frequency multiples which are detected in Fig. 1. For clarity the curves were shifted vertically by arbitrary amounts.

show frequency combs, see Fig. 3 of [32]. Since only total spectra were published [32], we recalculated the multichannel Fourier transform for Dictyostelium discoideum for each amino acid. Fig. 5 shows the total Fourier spectrum and the detailed spectra for some amino acids with important contributions towards the frequency comb. We confirmed that the frequency comb is the same type of frequency alias as seen for the genome of $P$. falciparum. For instance, glutamic acid (E) alone is sufficient to account for the $k / 28$ multiples of the power spectra presented by McLachlan et al. [32].

While nucleotide binary indicator mapping separates the symbolic genomic sequence into four binary sequences, they are separated into 20 sequences for amino acids. Therefore, the likelihood that an amino acid appears repeatedly and in isolation, is much higher than for nucleotides. It is therefore not surprising that for almost every work we encountered, power spectra in amino acid sequences were reported with multiple frequencies when binary mapping was used [7-9,32-35]. What remains somewhat of an open question is the absence of any explanation for the frequency multiples and fractional periodicities in all these works.

The presence of strong frequency aliases in the power spectra of so many chromossomes is presently unique to P. falciparum. However, considering the accelerated pace of genomic sequencing, we cannot rule out further occurrences of such frequency aliases in other genomes. In this work we stress the need for a careful evaluation of frequency multiples if they appear in Fourier power spectra since most available bioinformatics applications do not distinguish these aliases from genuine frequencies.

\section{Methods}

\section{Binary indicator sequence}

The original nucleotide sequence of length $N$ is converted into four numerical sequences of binary indicators,

$$
u_{\alpha, n} \begin{cases}1 & \text { if } \alpha \text { at } n \\ 0 & \text { otherwise }\end{cases}
$$

where the coefficient $u_{\alpha, n}$ indicates the presence or absence of a nucleotide of type $\alpha$ at position $n$ with 1 or 0 . This method is also sometimes called multichannel Fourier analysis where each $\alpha$ represents a channel [32].

For instance the sequence TAAGACCTATGTTAGTAAAG would be represented as shown in table 2 . This numerical representation removes any content bias of the original genomic sequences which may be an advantage or not depending on its intended applications $[18,36]$.

For a sequence of amino acids this mapping is trivially extended by mapping the 20 amino acids into the same amount of binary indicator sequences [32].

\section{Elastic constants}

An alternative to the binary indicator sequence is to convert the genomic DNA sequence into a single numerical sequence using a conversion table. In this work, we use microscopic flexibilities for DNA calculated recently [37]. Instead of converting single nucleotides we convert pair-wise nearest-neighbour dimers following table 3 . In this case the sequence 


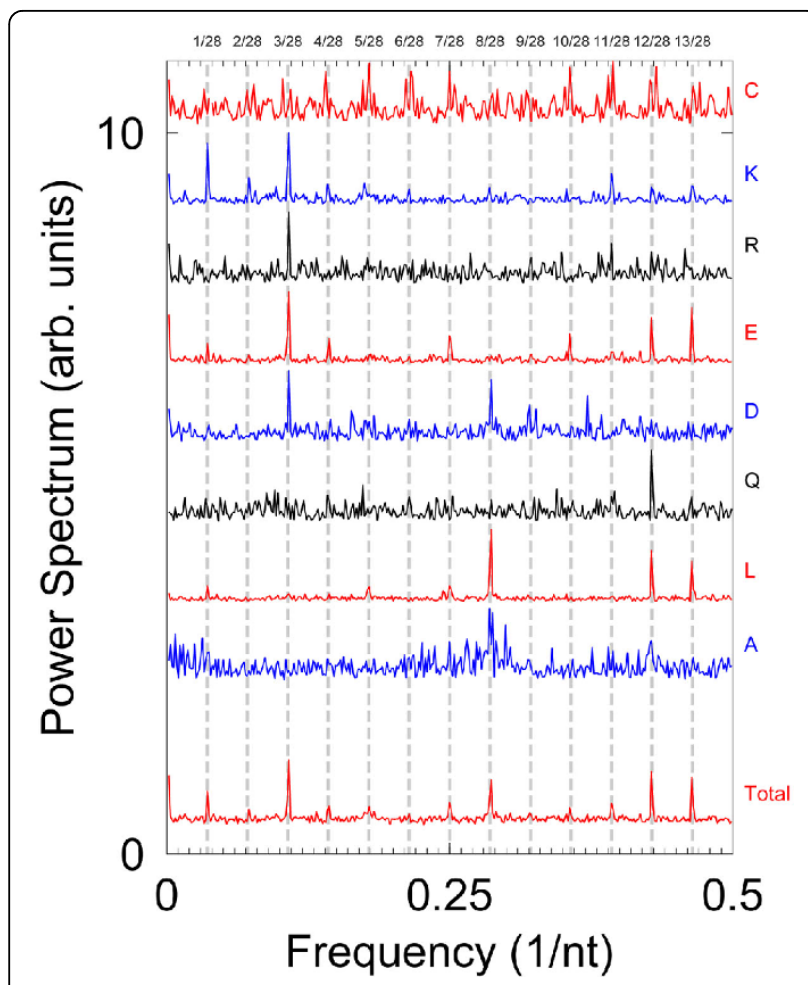

Figure 5 Binary indicator power spectra of Dictyostelium discoideum myosin heavy chain gene. In addition to the total spectrum, we show the individual spectra for amino acids with significant frequency harmonics. Vertical dashed lines are the positions of the $k / 28$ frequency multiples. The curves were shifted vertically by arbitrary amounts for clarity.

TAAGACCTATGTTAGTAAAG would be represented by the flexibility profile shown in figure 6 .

\section{Power spectrum}

For the binary indicator sequences we calculate the discrete Fourier transform (DFT) for each channel independently

$$
U_{\alpha, l}=\frac{1}{N} \sum_{n=0}^{N-1} u_{\alpha, n} e^{-i \frac{2 \pi}{N} l n}, l=0,1,2, \ldots, N-1
$$

where $N$ is the length of the sequence, and then combine the four resulting power spectra [24]

Table 3 Flexibilities of the ten unique nearest-neighbours dimers in DNA [37].

\begin{tabular}{llll}
\hline dimer step & $k\left(\mathrm{eV} / \mathrm{nm}^{2}\right)$ & dimer & $k\left(\mathrm{eV} / \mathrm{nm}^{2}\right)$ \\
\hline ApA & 2.40 & ApC & 2.56 \\
ApG & 2.25 & ApT & 1.83 \\
CpA & 3.44 & CpC & 2.06 \\
CpG & 2.74 & GpA & 2.80 \\
GpC & 3.36 & TpA & 2.42 \\
\hline
\end{tabular}

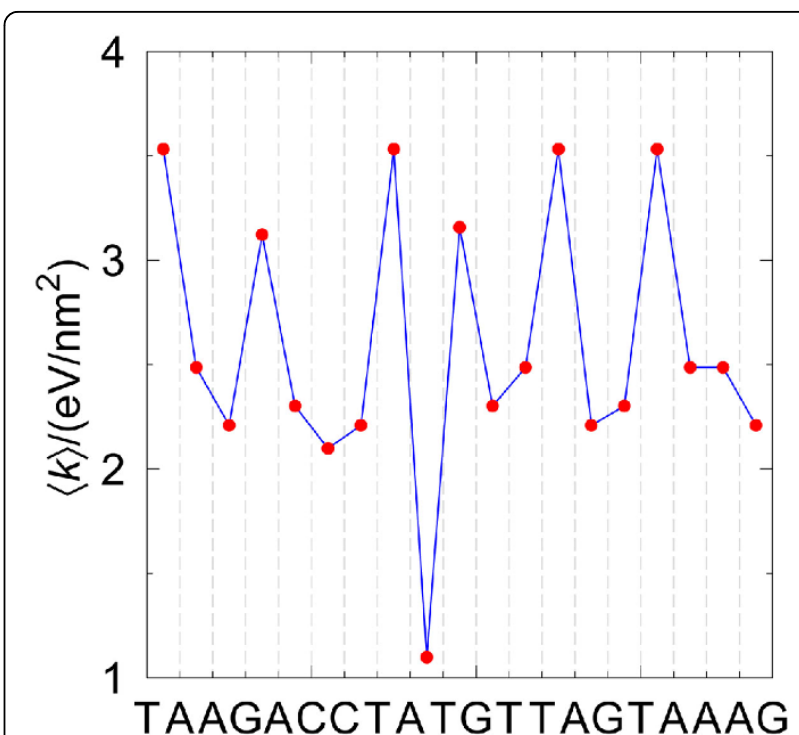

Figure 6 Example of flexibility profile. Each red bullet represents the flexibility of a given nearest-neighbour base-pairs of the sequence TAAGACCTATGTTAGTAAAG. For instance, the first is the flexibility of TpA, the second of ApA and so forth. Lines are intended as guide to the eye.

$$
S_{l}=\sum_{\alpha=\mathrm{A}, \mathrm{C}, \mathrm{G}, \mathrm{T}}\left|U_{\alpha, l}\right|^{2}
$$

The DFT was implemented through the use of the efficient FFTW3 package [38].

For the flexibility profile we calculate only one Fourier transform and its corresponding power spectra

$$
U_{l}=\frac{1}{M} \sum_{n=0}^{M-1} k_{n} e^{-i \frac{2 \pi}{M} l n}, S_{l}=\left|U_{l}\right|^{2}
$$

where $M$ is the length of the nearest-neighbour sequence and $M=N-1$.

\section{Position dependent power spectrum}

To identify the genomic origin of a given frequency $f$ we apply the Fourier transform only to a section of size $w$ starting at position $p$ of the genome and divide this section along the genome monitoring only this specific frequency. In this way we are able to pinpoint the genomic sections which provide the strongest contribution to a specific frequency $f=l / w$. For the case of binary indicators this means taking Eq. (2) only over a window $w$

$$
U_{\alpha, l}(p)=\frac{1}{w} \sum_{n=p}^{p+w} u_{\alpha, n} e^{-i \frac{2 \pi}{N} l n}, l=0,1,2, \ldots, N-1
$$


and then monitoring a specific frequency $f=l / w$. In this work we used a window of size $w=1000 \mathrm{nt}$, unless noted otherwise.

\section{Accession numbers}

In this work we used the genomic sequences of $P$. falciparum, accession numbers, in increasing order of chromosomes: NC_004325(1), NC_000910(2), NC_000521 (3), NC_004318(1), NC_004326(1), NC_004327(2), NC_004328(1), NC_004329(1), NC_004330(1), NC_004314(1), NC_004315(1), NC_004316(2), NC_004331(1), NC_004317(1). Version numbers are given in brackets. For Dictyostelium discoideum we used M14628(1).

\section{Author contribution}

MCSN wrote the Perl scripts, performed the calculations and prepared the figures. EFW and GW provided conceptual advice and supervised MCSN. All authors wrote the paper.

\section{Acknowledgements}

Funding CNPq, Capes and Fapemig.

This article has been published as part of BMC Genomics Volume 12 Supplement 4, 2011: Proceedings of the 6th International Conference of the Brazilian Association for Bioinformatics and Computational Biology ( $X$ meeting 2010). The full contents of the supplement are available online at http://www.biomedcentral.com/1471-2164/12?issue=S4

\section{Author details}

${ }^{1}$ Department of Biological Sciences, Federal University of Ouro Preto, 35400 000 Ouro Preto, MG, Brazil. ²Department of Computer Engineering, Federal Center for Technological Education of Minas Gerais, 30421-169 Belo Horizonte, MG, Brazil. ${ }^{3}$ Department of Physics, Federal University of Minas Gerais, 31270-901 Belo Horizonte, MG, Brazil.

\section{Competing interests}

The authors declare that they have no competing interests.

Published: 22 December 2011

\section{References}

1. Whiteford N, Haslam N, Weber G, Prügel-Bennett A, Essex JW, Roach PL Bradley M, Neylon C: An analysis of the feasibility of short read sequencing. Nucleic Acids Res 2005, 33(19):e171[http://nar.oxfordjournals. org/content/33/19/e171.abstract].

2. Vinces $M D$, Legendre $M$, Caldara $M$, Hagihara $M$, Verstrepen $K J$ : Unstable tandem repeats in promoters confer transcriptional evolvability. Science 2009, 324(5931):1213-1216[http://www.sciencemag.org/content/324/5931/ 1213.abstract].

3. Cordaux R, Batzer MA: The impact of retrotransposons on human genome evolution. Nat Rev Genet 2009, 10(10):691-703.

4. Treangen TJ, Abraham AL, Touchon M, Rocha EPC: Genesis, effects and fates of repeats in prokaryotic genomes. FEMS Microbiology Reviews 2009, 33(3):539-571

5. Huda A, Mariño-Ramírez L, Landsman D, Jordan IK: Repetitive DNA elements, nucleosome binding and human gene expression. Gene 2009, 436(1-2):12-22.

6. Whiteford N, Haslam N, Weber G, Prügel-Bennett A, Essex JW, Neylon C: Visualising the repeat structure of genomic sequences. Complex Systems 2008, 17:381-398.

7. Stewart M, McLachlan AD: Fourteen actin-binding sites on tropomyosin? Nature 1975, 257:331-333.
8. Parry DAD: Analysis of the primary sequence of a-tropomyosin from rabbit skeletal muscle. J. Mol. Biol 1975, 98:519-535.

9. McLachlan $A D$, Stewart M: The 14-fold periodicity in alpha-tropomyosin and the interaction with actin. J Math Biol 1976, 103(2):271-298[http:// www.sciencedirect.com/science/article/pii/0022283676903132].

10. Dowling LM, Crewther WG, Parry DA: Secondary structure of component $8 \mathrm{c}-1$ of alpha-keratin. An analysis of the amino acid sequence. Biochem J 1986, 236(3):705-712

11. Makeev VJu, Tumanyan VG: Search of periodicities in primary structure of biopolymers: a general Fourier approach. Comput Appl Biosci 1996, 12:49-54.

12. Veljković V, Cosić I, Dimitrijević B, Lalović D: Is it possible to analyze DNA and protein sequences by the methods of digital signal processing? IEEE Trans Biomed Eng 1985, 32(5):337-341.

13. Silverman BD, Linsker R: A measure of DNA periodicity. J Theor Biol 1986, 118(3):295-300[http://www.sciencedirect.com/science/article/pii/ S0022519386800601]

14. Benson DC: Fourier methods for biosequence analysis. Nucl. Acids. Res 1990, 18(21):6305.

15. Anastassiou D: Frequency-domain analysis of biomolecular sequences. Bioinformatics 2000, 16(12):1073.

16. Anastassiou D: Genomic signal processing. IEEE Signal Processing Mag 2001, 8-20.

17. Fukushima A, Ikemura T, Kinouchi M, Oshima T, Kudo Y, Mori H, Kanaya S: Periodicity in prokaryotic and eukaryotic genomes identified by power spectrum analysis. Gene 2002, 300:203-211.

18. Akhtar M, Epps J, Ambikairajah E: Signal processing in sequence analysis: advances in eukaryotic gene prediction. IEEE Journal on Selected Topics in Signal Processing 2008, 2(3):310-321.

19. Gardner MJ, Hall N, Fung E, White O, Berriman M, Hyman RW, Carlton J, Pain A, Nelson K, Bowman $S$, et al: Genome sequence of the human malaria parasite Plasmodium falciparum. Nature 2002, 419(6906):498-511.

20. Wicker T, Sabot F, Hua-Van A, Bennetzen JL, Capy P, Chalhoub B, Flavell A, Leroy $P$, Morgante $M$, Panaud $O$, et al: A unified classification system for eukaryotic transposable elements. Nature Reviews Genetics 2007, 8(12):973-982.

21. Sharma D, Issac B, Raghava GPS, Ramaswamy R: Spectral Repeat Finder (SRF): identification of repetitive sequences using Fourier transformation. Bioinformatics 2004, 20(9):1405-1412.

22. Brodzik A: Quaternionic periodicity transform: an algebraic solution to the tandem repeat detection problem. Bioinformatics 2007, 23(6):694.

23. Epps J: A hybrid technique for the periodicity characterization of genomic sequence data. EURASIP J Bioinform Syst Biol 2009, 924601.

24. Tiwari S, Ramachandran S, Bhattacharya A, Bhattacharya S, Ramaswamy R: Prediction of probable genes by Fourier analysis of genomic sequences. Comput. Appl. Biosci 1997, 13(3):263-270.

25. Issac B, Singh H, Kaur H, Raghava GPS: Locating probable genes using Fourier transform approach. Bioinformatics 2002, 18:196-197[http:// bioinformatics.oxfordjournals.org/content/18/1/196.abstract].

26. Kotlar D, Lavner Y: Gene prediction by spectra rotation measure: a new method for identifying protein-coding regions. Genome Res 2003, 13:1930-1937.

27. Gao J, Qi Y, Cao Y, Tung WW: Protein coding sequence identification by simultaneously characterizing the periodic and random features of DNA sequences. J Biomed Biotechnol 2005, 2005:139-146.

28. Benson G: Tandem repeats finder: a program to analyze DNA sequences. Nucleic Acids Res 1999, 27(2):573-580[http://nar.oxfordjournals.org/content/ 27/2/573.abstract].

29. Brigham EO: The fast Fourier transform and its applications. London: Prentice-Hall International; 1988.

30. Lobzin W, Chechetkin VR: Order and correlations in genomic DNA sequences. The spectral approach. Physics-Uspekhi 2000, 43:55-78.

31. Wang L, Schonfeld D: Mapping Equivalence for Symbolic Sequences: Theory and Applications. IEEE Transactions on Signal Processing 2009, 57(12):4895-4905.

32. McLachlan AD: Multichannel Fourier analysis of patterns in protein sequences. J. Phys. Chem 1993, 97(12):3000-3006.

33. McLachlan AD, Karn J: Periodic features in the amino acid sequence of nematode myosin rod. J. Mol. Biol 1983, 164(4):605-626.

34. Taylor WR, Heringa J, Baud F, Flores TP: A Fourier analysis of symmetry in protein structure. Protein Eng 2002, 15(2):79-89. 
35. Gruber M, Soding J, Lupas AN: REPPER-repeats and their periodicities in fibrous proteins. Nucl. Acids. Res 2005, 33(Web Server Issue):W239.

36. Paar V, Pavin N, Basar I, Rosandić M, Glunčić M, Paar N: Hierarchical structure of cascade of primary and secondary periodicities in Fourier power spectrum of alphoid higher order repeats. BMC Bioinformatics 2008, 9:466

37. Weber G, Essex JW, Neylon C: Probing the microscopic flexibility of DNA from melting temperatures. Nature Physics 2009, 5:769-773.

38. Frigo M, Johnson SG: The Design and Implementation of FFTW3. Proceedings of the IEEE 2005, 93(2):216-231, Special issue on "Program Generation, Optimization, and Platform Adaptation".

doi:10.1186/1471-2164-12-S4-S4

Cite this article as: Nunes et al.: Origin of multiple periodicities in the

Fourier power spectra of the Plasmodium falciparum genome. BMC

Genomics 2011 12(Suppl 4):S4.

Submit your next manuscript to BioMed Central and take full advantage of:

- Convenient online submission

- Thorough peer review

- No space constraints or color figure charges

- Immediate publication on acceptance

- Inclusion in PubMed, CAS, Scopus and Google Scholar

- Research which is freely available for redistribution

Submit your manuscript at www.biomedcentral.com/submit 